\title{
REPARACIÓN LAPAROSCÓPICA DE FÍSTULA VESICOVAGINAL MEDIANTE TÉCNICA RETROVESICAL: UNA SERIE DE 6 PACIENTES
}

\author{
Marcelo Kerkebe L. ${ }^{1,2}$, Enrique Bley V. ${ }^{2}$, Pablo Pizzi L. ${ }^{1}$, Cristian Falcón B. ${ }^{2}$, Carlos \\ Iturriaga V. ${ }^{2}$, Walter Candia G. ${ }^{2}$, Fernando Heredia M. ${ }^{3}$ \\ 1 Unidad de Urología, Clínica Tabancura. ${ }^{2}$ Departamento de Urología, Facultad de Medicina, Hospital del Salvador, \\ Universidad de Chile. ${ }^{3}$ Departamento de Ginecología y Obstetricia, Facultad de Medicina, Universidad de Concepción.
}

\section{RESUMEN}

Antecedentes: La fístula vesicovaginal (FVV) es una enfermedad frecuente en países no desarrollados y afecta la calidad de vida de mujeres en edad media, siendo la causa más habitual la histerectomía previa. Las técnicas quirúrgicas disponibles para su reparación presentan resultados variables. Objetivos: Presentar nuestra experiencia en la reparación de FVV por vía laparoscópica con abordaje retrovesical, en una serie consecutiva de 6 pacientes. Método: análisis prospectivo descriptivo de 6 pacientes sometidos a reparación laparoscópica retrovesical de FVV supratrigonales secundarias a histerectomías. Resultados: EI tiempo operatorio promedio fue de 191 minutos. Ningún paciente requirió transfusiones y el tiempo de hospitalización promedio fue de 2,5 días. No se presentaron complicaciones ni recidivas, con un seguimiento promedio de 15 meses. Conclusión: La reparación laparoscópica de FVV mediante técnica retrovesical es una técnica segura, poco invasiva y reproducible en manos entrenadas, que podría convertirse en la técnica de elección a futuro. De acuerdo a nuestra revisión, esta comunicación es la primera serie de reparación laparoscópica de FVV publicada en Chile.

\section{PALABRAS CLAVE: Laparoscopia, fístula vesicovaginal, cirugía reconstructiva}

\section{SUMMARY}

Background: Vesicovaginal fistula (VVF) is a fairly common condition in underdeveloped countries affecting mainly young women. Its most prevalent cause is prior hysterectomy. Surgical techniques for repairing VVF have widespread results. Objective: To present our results with a retrovesical laparoscopical repair of VVF in a 6 consecutive patient case series. Method: It is a descriptive prospective analysis of 6 consecutive patients with a retrovesical laparoscopical repair of supratrigonal VVF after hysterectomy. Results: Average surgical time was 191 minutes. No blood transfusion was required. Average hospital stay was 2.5 days. None of them had postoperatory complications nor relapse whatsoever, after a minimum of 15 months follow-up. Conclusion: Retrovesical laparoscopic repair of VVF is a feasible, simple, reproducible and less invasive technique. In trained hands it is the election technique for this condition. To the best of our knowledge this is the first publication of laparoscopic management for VVF in Chile.

KEY WORDS: Laparoscopy, vesicovaginal fistula, reconstructive surgery 


\section{INTRODUCCIÓN}

La fístula vesicovaginal (FVV) es una patología de alta prevalencia en países subdesarrollados, existiendo actualmente en África más de 3.000 .000 de mujeres portadoras de FVV declarada irreparables, con una incidencia anual de 130.000 nuevos casos (1). Es además una patología que afecta a mujeres de edad media provocando un deterioro severo en la calidad de vida de quien las padece (2).

Si bien la FVV presenta múltiples causas, tales como complicaciones de la radioterapia pélvica, neoplasias, accidentes obstétricos, aborto ilegal y otras, actualmente la principal etiología es la complicación iatrogénica de la histerectomía (3). Un $62-87 \%$ de las FVV que se diagnostican a nivel mundial se presentan como complicación de esta cirugía, siendo la edad media de presentación los 52 años (1).

Las técnicas para reparación quirúrgica disponibles nos entregan resultados variables a largo plazo, con recidivas que van desde el 4 a $37 \%$ (25). Dependiendo de la ubicación, el abordaje quirúrgico puede ser transvaginal (fístulas trigonales) o abdominal (fístulas supratrigonales). Estas últimas son más frecuentes como consecuencia de histerectomías. La técnica de reparación más difundida es la transvesical, descrita por O Conor (6).

La cirugía laparoscópica ha tenido un desarrollo exponencial en los últimas 2 décadas, debido a sus ventajas en el intra y pos operatorio sobre la cirugía convencional, pudiendo ser aplicada en la actualidad en la mayoría de los procedimientos quirúrgicos.

El objetivo de esta comunicación es presentar nuestra experiencia de 6 pacientes con FVV supratrigonal resueltas mediante abordaje laparoscópico retrovesical.

\section{MATERIAL Y MÉTODO}

Análisis prospectivo descriptivo de 6 pacientes portadoras de FVV supratrigonal reparadas por vía laparoscópica en forma consecutiva por el mismo cirujano (MK), entre marzo de 2006 y mayo de 2008. El promedio de edad de las pacientes fue de 45,5 años (rango: 41 -51 años), en todos los casos fue secundario a histerectomía por vía abdominal. Solo una paciente tenía antecedentes de cirugía abdominal previa (colecistectomía). Las características de las pacientes que integraron esta serie se detallan en la Tabla I. Se evaluó tiempo operatorio total (tiempo cistoscópico más laparoscópi$\mathrm{co}$ ), magnitud del sangrado, complicaciones intra y post operatorias, tiempo de hospitalización, tiempo de sonda Foley y evolución. En todos los casos la cirugía realizada se trató de una reparación laparoscópica por vía retrovesical, según técnica que se describe a continuación.

Técnica quirúrgica: Anestesiada la paciente se posiciona en decúbito dorsal con ambos brazos adosados al cuerpo y con ambas piernas separadas y semi flexionadas de tal manera de tener acceso al área genital.

Tabla I

ANTECEDENTES CLÍNICOS DE LAS PACIENTES

\begin{tabular}{|c|c|c|c|c|c|c|}
\hline Caso & $\begin{array}{l}\text { Peso } \\
(\mathrm{kg})\end{array}$ & $\begin{array}{l}\text { Talla } \\
(\mathrm{cm})\end{array}$ & Patología & $\begin{array}{c}\text { Tiempo } \\
\text { de fistulización }\end{array}$ & $\begin{array}{c}\text { Tipo } \\
\text { de fístula }\end{array}$ & $\begin{array}{c}n^{\circ} \\
\text { de fístulas }\end{array}$ \\
\hline 1 & 80 & 153 & Miomatosis uterina & 10 meses & Supratrigonal & 2 \\
\hline 2 & 65 & 173 & CACU & 1 mes & Supratrigonal & 1 \\
\hline 3 & 85 & 150 & Cistoadenoma ovárico & 2 meses & Supratrigonal & 1 \\
\hline 4 & 72 & 150 & Miomatosis uterina & 1 mes & Supratrigonal $+\mathrm{P}$ & 2 \\
\hline 5 & 70 & 156 & CACU & 3 meses & Supratrigonal & 1 \\
\hline 6 & 55 & 145 & Miomatosis uterina & 18 meses & Supratrigonal & 1 \\
\hline
\end{tabular}

CACU: cáncer cervicouterino. P: peritoneal. 


\section{Tiempo cistoscópico:}

a. Cistoscopia convencional con el fin de identificar los orificios ureterales y el orificio fistuloso.

b. Cateterización de orificios ureterales.

c. Por vía transvaginal, canulación del trayecto de la fístula con pinza Randall de ángulo recto.

d. Por vía vesical, se enhebra catéter ureteral, de diferente color a los utilizados en los uréteres, con ayuda de pinza Randall, la que se exterioriza por vagina (Figura 1).

\section{Tiempo laparoscópico:}

a. Neumoperitoneo abierto a nivel umbilical. Se instalan 5 trócares en forma de $\mathrm{V}$ invertida con vértice en el ombligo.

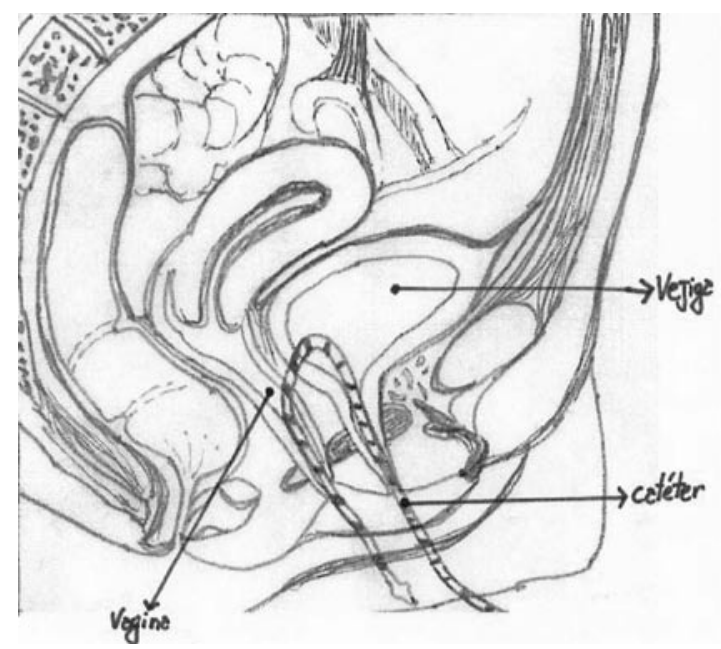

Figura 1. Visión sagital de catéter insertado por vejiga conectado con vagina. b. Disección del plano vesicovaginal hasta visualizar el catéter ureteral trans fistuloso (Figura 2).

c. Disección de orificios fistulosos vesical y vaginal (Figura 3).

d. Reparación de defectos con vicryil 3/0, con puntos corridos intracorpóreos, un plano a la vagina y 2 planos a la vejiga (Figura 4).

e. Interposición de epiplón entre pared posterior de vejiga y pared anterior de vagina si es técnicamente factible.

f. Tacto vaginal para comprobar correcta reparación vaginal.

g. Cistoscopia para comprobar correcta reparación vesical.

h. Instalación de drenaje.

\section{RESULTADOS}

La media del tiempo operatorio fue de $191 \mathrm{mi}-$ nutos (rango: 160-210 minutos). No hubo necesidad de transfusión. El promedio de tiempo de sonda vesical fue 20,8 días sin necesidad de realizar un uretrocistografía previa. No hubo complicaciones intra o pos operatorias. No hubo conversiones a cirugía abierta. Los detalles de las intervenciones se resumen en la Tabla II. El seguimiento promedio fue de 15 meses, no existiendo hasta el momento pacientes que presenten recidiva o complicaciones tardías.
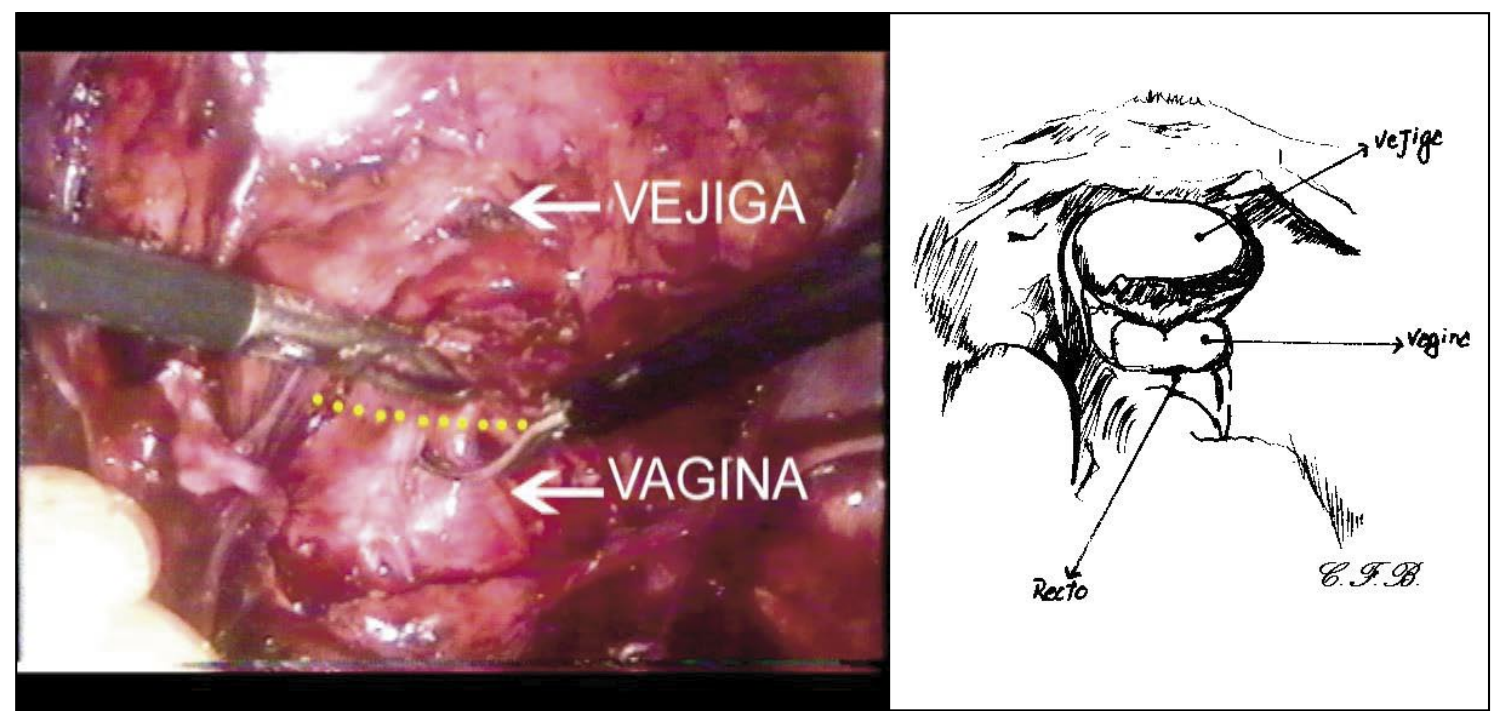

Figura 2. Plano de disección vesicovaginal que muestra la visión laparoscópica. 

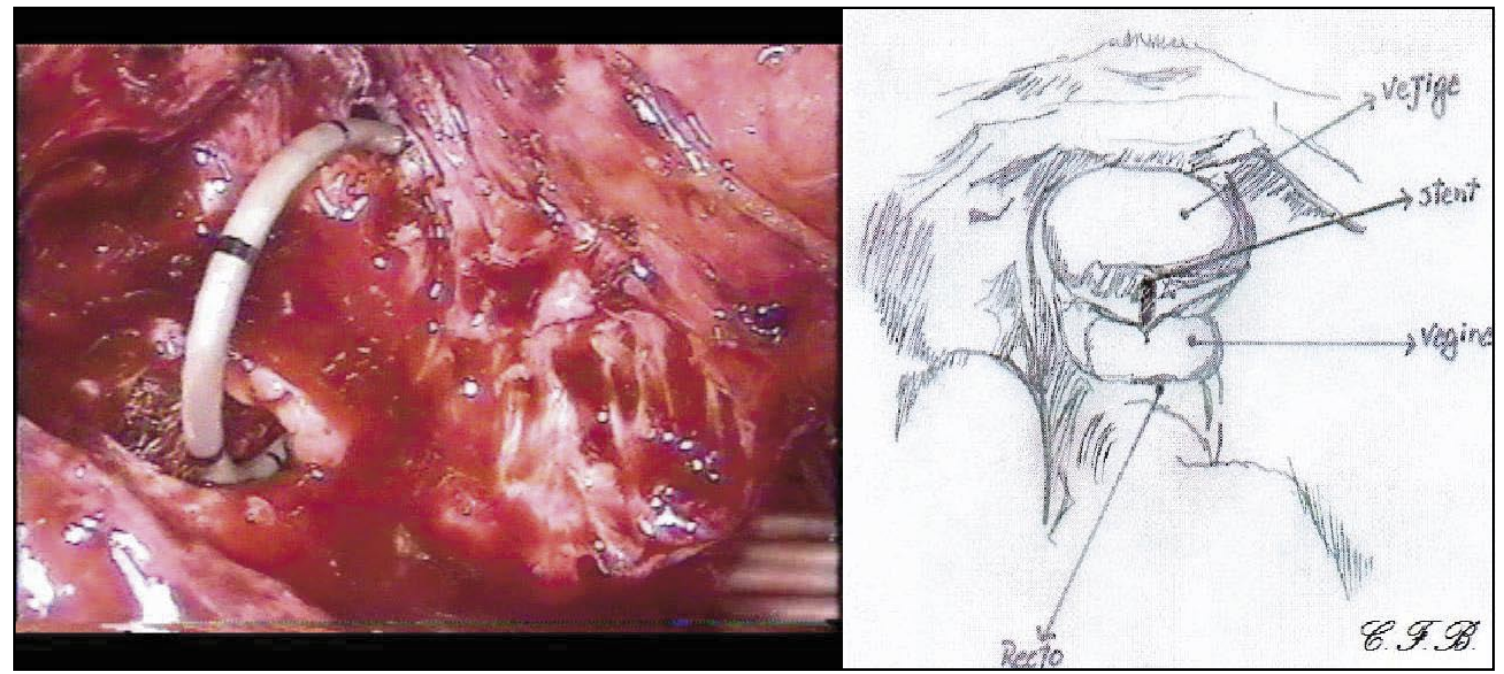

Figura 3. Visión laparoscópica que muestra el Stent que comunica vejiga y vagina.
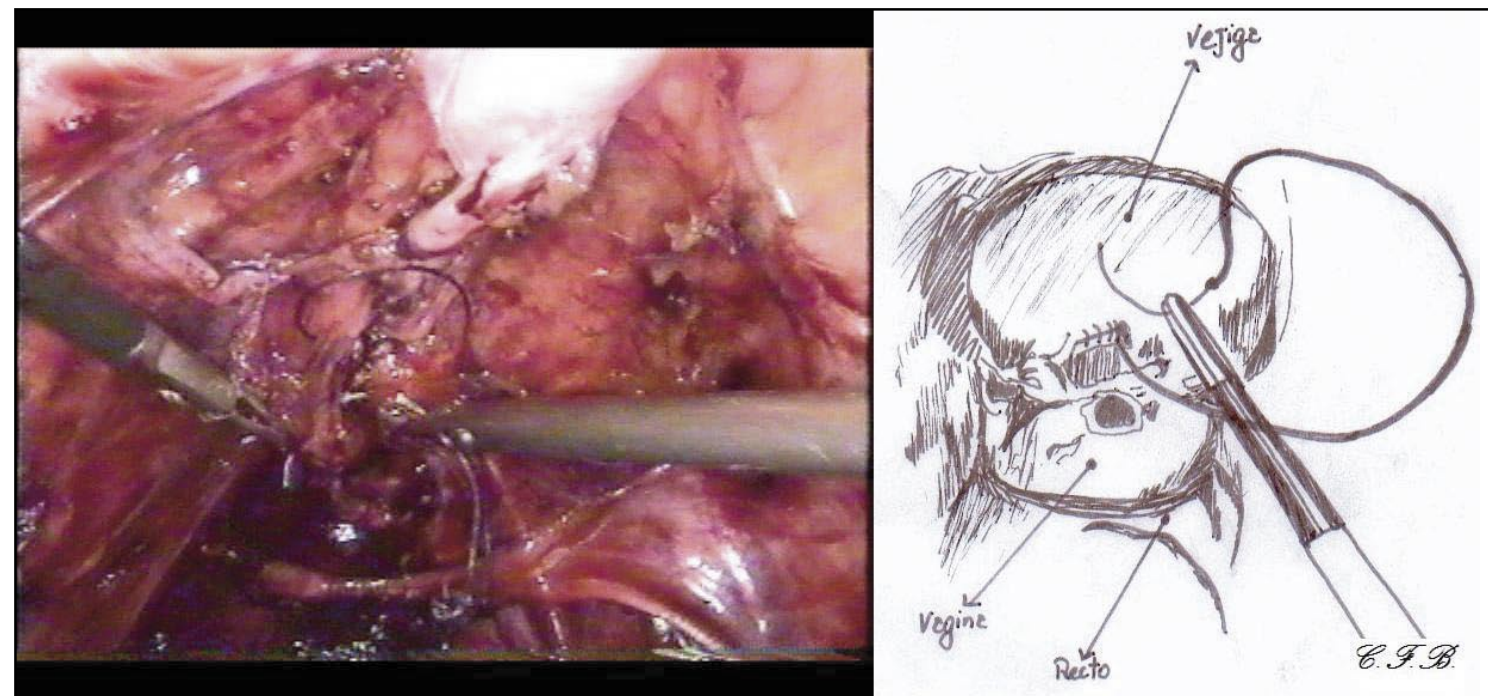

Figura 4. Visión laparoscópica que muestra la sutura de la comunicación.

Tabla II

TIEMPO OPERATORIO, VOLUMEN DE SANGRADO, DÍAS DE HOSPITALIZACIÓN Y TIEMPO DE USO DE SONDA VESICAL

\begin{tabular}{ccccc}
\hline Caso & $\begin{array}{c}\text { Tiempo operatorio } \\
\text { (minutos) }\end{array}$ & $\begin{array}{c}\text { Volumen del sangrado } \\
(\mathrm{cc})\end{array}$ & $\begin{array}{c}\text { Hospitalización } \\
\text { (días) }\end{array}$ & $\begin{array}{c}\text { Tiempo de sonda } \\
\text { (días) }\end{array}$ \\
\hline 1 & 200 & 100 & 3 & 20 \\
2 & 210 & 100 & 2 & 21 \\
3 & 210 & 100 & 2 & 21 \\
4 & 160 & 200 & 3 & 21 \\
5 & 210 & 150 & 3 & 21 \\
6 & 160 & 50 & 2 & 21 \\
\hline
\end{tabular}




\section{DISCUSIÓN}

Los conceptos principales que persiguen cualquier técnica de reparación quirúrgica de una fístula, no han cambiado demasiado desde las primeras recomendaciones publicadas en 1852: evaluación preoperatorio adecuada, buena exposición de la fístula, resección del tejido fibroso o desvitalizado, suturas libres de tensión y un buen drenaje por un tiempo adecuado (8).

La técnica retrovesical de reparación laparoscópica para FVV que se ha descrito permite respetar a cabalidad estos principios. El abordaje retrovesical es dificultoso por vía abierta ya que la visión de la cara posterior de la vejiga y la cara anterior de la vagina es limitada. Por ello la técnica de O'Connor es de elección en el abordaje convencional (6). Por el contrario, la disección de este plano por vía laparoscópica está facilitada por el uso de la óptica de $30^{\circ}$ que permite la exposición total de dichas superficies sin la necesidad de la realización de cistotomía, lo que se traduce en un menor sangrado, recuperación más rápida y en un menor tiempo de sondeo vesical (9-10). En nuestra experiencia inicial preferimos no adelantarnos en el retiro de la sonda Foley para asegurarnos un cierre definitivo de la fístula.

Nuestra serie confirma la prevalencia de esta complicación en mujeres en la $4^{\mathrm{a}}$ y $5^{\mathrm{a}}$ década de la vida, que ven disminuida su actividad social, laboral y sexual por esta complicación, llegando al momento de la cirugía con un gran deterioro psico-físico. Es aquí donde vemos reflejada la gran ventaja del abordaje laparoscópico ya que brinda una rápida recuperación en el pos operatorio, con mínimo malestar, deambular y alta precoz. Esto, junto con los resultados estéticos que brinda una cirugía mínimamente invasiva, hace que este procedimiento tenga un gran impacto en la recuperación anímica de las pacientes.

Cabe destacar que la cirugía laparoscópica reconstructiva es de alta complejidad, a ello debemos sumarle un ambiente de cirugía previa que se traduce en adherencias, y un tejido friable secundario a la inflamación crónica. Por lo anterior esta cirugía debe ser realizada por cirujanos con experiencia en cirugía laparoscópica reconstructiva, con un adecuado manejo de la sutura intracorpórea.

Los resultados obtenidos en esta serie son comparables a series similares publicadas en la literatura, destacando en la nuestra, una reducción en los tiempos operatorios, el sangrado, los días de hospitalización, las complicaciones y los resultados a largo plazo, al compararnos con series similares de reparación laparoscópica pura y asistida por robot (9-17) (Tabla III).

\section{CONCLUSIÓN}

La reparación retrovesical laparoscópica de la FVV es una alternativa segura, poco invasiva y reproducible en manos entrenadas. Si bien nuestra experiencia al igual que la mundial aún es incipiente, creemos que pronto se convertirá en el abordaje de elección para la reparación de FVV supratrigonales. Según nuestra revisión esta es la primera serie de reparación laparoscópica de FVV publicada en Chile.

Tabla III

CUADRO COMPARATIVO CON OTRAS SERIES

\begin{tabular}{lccccc}
\hline Variable & Abreu $^{14}$ & Sundaram $^{16}$ & Otsuka $^{17}$ & Sotelo $^{18}$ & Kerkebe $^{18}$ \\
\hline N $^{\circ}$ casos & 8 & 5 & 7 & 15 & 6 \\
N $^{\circ}$ casos retrovesical & - & 0 (robot) & 2 & 0 & 6 \\
Recurrencia & 1 & 0 & 0 & 1 & 0 \\
Transfusión & 1 & 0 & - & - & 0 \\
Complicación & 1 & 0 & 2 & 170 & 0 \\
Tiempo operatorio (minutos) & - & 233 & 280 & $140-240$ & $160-210$ \\
Tiempo operatorio: rango & $170-390$ & $150-330$ & $130-420$ & - & 116 \\
Sangrado (mL) & - & 70 & - & 3 & 2,5 \\
Hospitalización (días) & - & 5 & 7,2 & 10,2 & 20,8 \\
Cateter vesical (días) & - & 10 & 30 & & \\
\hline
\end{tabular}




\section{BIBLIOGRAFÍA}

1. Wall LL. Obstetric vesicovaginal fistula as an international public-health problem. Lancet 2006;368(9542):1201-9.

2. Cohen BL, Gousse AE. Current techniques for vesicovaginal fistula repair: Surgical pearls to optimize cure rate. Curr Urol Rep 2007;8(5):413-8.

3. Creanga AA, Genadry RR. Obstetric fistulas: a clinical review. Int J Gynaecol Obstet 2007;99 Suppl 1:S40-6. Epub 2007 Sep 14.

4. Kochakarn W, Pummangura W. A new dimension in vesicovaginal fistula management: an 8-year experience at Ramathibodi hospital. Asian $\mathrm{J}$ Surg 2007;30(4):267-71.

5. Vallancien G, Cathelineau X, Baumert H, Doublet JD, Guillonneau B. Complications of transperitoneal laparoscopic surgery in urology: review of 1,311 procedures at a single center. J Urol 2002;168(1): 23-6.

6. Nesrallah LJ, Srougi M, Gittes RF. The O Conor technique: the gold standard for supratrigonal vesicovaginal fistula repair. J Urol 1999;261:566-8.

7. Fahlenkamp D, Rassweiler J, Fornara P, Frede T, Loening SA. Complications of laparoscopic procedures in urology: experience with 2,407 procedures at 4 German centers. J Urol 1999;162(3):765-71.

8. Hamilton JN, Rovner ES, Turner WR. Urology in precivil war Charleston. J Urol 2008;180(2):477-80.

9. Modi P, Goel R, Dodia S. Laparoscopic repair of vesicovaginal fistula. Urol Int 2006;76(4):374-6.
10. Erdogru T, Sanli A, Celik O, Baykara M. Laparoscopic transvesical repair of recurrent vesicovaginal fistula using with fleece-bound sealing system. Arch Gynecol Obstet 2008;277(5):461-4.

11. Gerber GS, Rukstalis DB, Levine LA, Chodak GW. Current and future roles of laparoscopic surgery in urology. Urology 1993;41(1 Suppl):5-9.

12. Schimpf MO, Morgenstern JH, Tulikangas PK, Wagner JR. Vesicovaginal fistula repair without intentional cystotomy using the laparoscopic robotic approach: a case report. JSLS 2007;11(3):378-80.

13. Dos Santos Abreu L de A, Tanaka M, de Abreu SC, Kawano PR, Yamamoto H, Otsuka RA, Marcelo Rosa Travassos MR, Amaro JL, Fugita OE. Laparoscopic management of iatrogenic lesions. J Endourol 2008;22(6):1279-83.

14. Melamud O, Eichel L, Turbow B, Shanberg A. Laparoscopic vesicovaginal fistula repair with robotic reconstruction. Urology 2005;65(1): 163-6.

15. Sundaram BL, Kalidasan G, Hemal AK. Robotic repair of vesicovaginal fistula: case series of five patients. Urology 2006;67(5): 970-3.

16. Otsuka RA, Amaro JL, Tanaka MT, Epacagnan E, Mendes JB Jr, Kawano PR, Fugita OE. Laparoscopic repair of vesicovaginal fistula. J Endourol 2008;22(3):525-7.

17. Sotelo R, Mirandolino M, García-Segui A, Dubois R, Spaviliero M, Keklikian W, Novoa J, Yaime H, Finelli A. Laparoscopic repair of vesicovaginal fistula. J Urol 2005;173(5):1615-8. 